\title{
BMJ Open Exploring educational needs and design aspects of internet-enabled patient education for persons with diabetes: a qualitative interview study
}

\author{
Javad Jafari, ${ }^{1,2}$ Hosein Karimi Moonaghi, ${ }^{3,4,5}$ Nabil Zary, ${ }^{1,6}$ Italo Masiello ${ }^{7}$
}

To cite: Jafari J, Karimi Moonaghi $\mathrm{H}$, Zary N, et al. Exploring educational needs and design aspects of internet-enabled patient education for persons with diabetes: a qualitative interview study. BMJ Open 2016;6:e13282.

doi:10.1136/bmjopen-2016013282

- Prepublication history for this paper is available online. To view these files please visit the journal online (http://dx.doi.org/10.1136/ bmjopen-2016-013282).

Received 7 July 2016 Revised 22 August 2016 Accepted 29 September 2016

CrossMark

For numbered affiliations see end of article.

Correspondence to Dr Javad Jafari; javad.jafari@ki.se

\section{ABSTRACT}

Objective: The objective of this article is to explore the educational needs and design aspects of personalised internet-enabled education for patients with diabetes in Iran.

Design: Data were collected using semistructured interviews and then qualitatively analysed using inductive content analysis.

Participants: 9 patients with type 2 diabetes were included. Inclusion criteria were access to and knowledge on how to use the internet. The selection ensured representation based on gender, age, occupation and educational background.

Setting: The sample population was patients with diabetes who were admitted to an outpatient diabetes clinic in Mashhad, a large city of Iran with about 3 million inhabitants.

Results: 4 core categories emerged from the data: (1) seeking knowledge about diabetes, including specific knowledge acquisition, patient's interactions and learning requirements; (2) teaching and learning, including using different teaching methods and different ways to learn about the disease; (3) facilitators, including internet and mobile phone use to learn about the disease; and (4) barriers, including lack of internet access, uncertainty of access to the internet and lack of website in the local language and also perceived cultural barriers, such as patients' fears of the internet, lack of time and awareness.

Conclusions: This study provides a better understanding of the patient's educational expectations and technical needs in relation to internet-enabled education. This knowledge will inform the development of functional mock-ups in the next research phase using a design-based research approach in order to design internet-enabled patient education for selfmanagement of diabetes.

\section{INTRODUCTION}

The International Diabetes Federation ${ }^{1}$ estimates that the global population of patients with diabetes mellitus (DM) today exceeds 415 million people. Having doubled

\section{Strengths and limitations of this study}

- Use of a design-based research approach to study needs-oriented internet-enabled education for patients with type-2 diabetes.

- This study provides empirical evidence on patients' educational needs and different ways to learn about their disease.

- This study also identifies facilitators and barriers to the effective use of internet-enabled patient education.

- The sample group was relatively small and came from one diabetes clinic, but our results were relevant to middle-income countries and more specifically to countries with a similar health culture and internet infrastructure.

worldwide from 1980 to 2008, it is estimated that the number of patients with DM will increase to more than 550 million by the year 2030, making diabetes as the seventh leading cause of death. ${ }^{2}$ About $80 \%$ of all patients with DM live in low and middle income countries, with most of them between the ages of 40 and 60 years. ${ }^{3}$ Type 2 diabetes (T2D) is the most prevalent form of diabetes, and in high income countries, up to $91 \%$ of adults with DM have T2D. ${ }^{1}$

There is currently no cure for diabetes, but effective treatments exist. ${ }^{4}$ Medications to lower the glucose level, including insulin, together with quality of care, food intake, physical activity levels and good medical advice can help patients with DM lead an active and healthy life and can reduce the risks of developing complications. Daily physical activity is recommended and, together with weight loss, can improve insulin resistance and an optimal level of blood glucose and lipids while reducing blood pressure. ${ }^{5}$ Lifestyle interventions planned to influence an individual's physical activity levels and diet are critical areas of T2D management. ${ }^{6}$ 


\section{Patient education}

Patient education is an important factor for enabling patients with DM to successfully take care of their health, ${ }^{7}$ and patients with DM need structured highquality education and support to develop their self-care. ${ }^{8}$ Patient education aims to improve health by encouraging compliance with medical treatment regimens and promoting healthy lifestyles. However, the value of selfmonitoring blood glucose by patients with T2D has mixed outcomes. ${ }^{9} 10$ Research suggests that selfmonitoring does not improve glycaemic control and may even increase anxiety. ${ }^{9}$ Qualitative studies report that self-monitoring is perceived to have negative impact on quality of life when identified problems in the monitoring of blood glucose levels are not taken care of quickly. ${ }^{11}$ Whereas, when clinical information is timely shared with the healthcare provider blood glucose control is improved in patients with T2D. ${ }^{12}$ Research has shown that patients who use self-monitoring techniques, including monitoring food intake, physical activity and glucose levels, have better control of their disease, ${ }^{13}$ even though a recent UK study suggests that the improvement of blood glucose control may not be large enough to be clinically relevant. ${ }^{14}$ Therefore, the acceptability of self-monitoring in patient with T2D is unclear. Nonetheless, several successful educational programmes have focused on empowerment of patients with DM. ${ }^{15}$

\section{Technology-enabled patient education}

New technology, such as mobile phones and web-based services (ie, internet-enabled) are inexpensive and convenient means of communication and their use has increased, even in middle and low income countries. ${ }^{16}$ This widespread distribution of mobile phones, across socioeconomic, gender, and age groups, combined with their unique ability to communicate data in real-time makes them an ideal platform to create internet-enabled services for real-time diabetes management programmes. ${ }^{17}$ Despite the huge numbers of diabetes applications (apps), most offer a small number of similar functionalities. In addition, patients are not involved during the app development, which excludes important patients' needs. ${ }^{18}$ Online portals and apps are convenient, cost-effective and easy to use anywhere at any time to know more about diabetes and how to individualise and manage self-care. ${ }^{19}$

According to the statistics provided by the Internet World Stats in November 2015, more than half of the Iranian population $(57.2 \%)$ used the internet, while in $2000, \sim 0.5 \%$ used it. This shows a rapid growth of internet use by the Iranian population. ${ }^{20}$ In our previous study, we found that $26.5 \%$ of a patient with DM had routine access to the internet, and $77.8 \%$ of those were positive about using an official Iranian website for medical information. However, $55 \%$ of the respondents still preferred to obtain health-related information from television, radio and educational films. ${ }^{21}$ Both technology use $^{19}$ and the incidence of diabetes ${ }^{6}$ have been increasing in Iran. It has become of national and general interest to bring forward research on internet-enabled patient education to determine future self-care management patterns of T2D.

The objective of this study is to explore the educational needs and design features of personalised internet-enabled education of a sample of patients with DM in Iran as an example of a middle-income country. The research questions we address are: what are the educational needs for self-care management of patients with T2D? Which structure, components and features of a technology-based instructional design should be included in a system for self-care management?

\section{METHODS}

\section{Study design}

The design-based research framework was adopted to guide the researchers to ultimately improve educational practice through better design of technology-enhanced learning environments. ${ }^{22}$ A qualitative approach was then used to explore the objective of this study. The researchers used inductive content analysis to provide a systematic and objective means of describing the educational needs and design features of personalised webbased and mobile phone-based education among the studied patients with DM population. ${ }^{23}$ Semistructured and personal interviews were conducted. We followed an in-depth protocol to further investigate a number of subjects introduced by the participants. ${ }^{24}$ The interviews were tape recorded and transcribed verbatim, and every interview lasted between 30 and $45 \mathrm{~min}$. The interviews were conducted in Persian by the first author, while the analysis of the interview data was conducted by two of the authors and translated into English.

\section{Participants and context of study}

The sample population consisted of nine patients with DM admitted to an outpatient diabetes clinic in Mashhad, a large city in Iran with about three million inhabitants. All interviews were conducted in a separate room of the diabetes clinic and at the participant's convenience with regard to time. About 7000 patients annually visited the diabetes clinic for care and check-up. A number of patients fitting the study's criteria were asked to participate in the project when they came to the clinic for routine check-up. Only one patient who refused to sign the consent form withdrew from the study. The interviewer was a medical doctor and $\mathrm{PhD}$ student who had worked in the clinic for about 6 years; he had experience with both patients with DM and the clinical environment and also he was trained on how to conduct qualitative research by undertaking courses during the PhD study period.

Participants were selected based on purposive sampling that considered gender, age, occupation and educational background. All participants signed a consent form prior to the interview. Participants were recruited 
on the basis of willingness to participate in the study, confirmed diagnosis of T2D at least 1 year prior to the study, had access to the internet at home or in the work place, had more than 9 years of formal education and were cognitively and physically able to participate personally in the study. To enhance the rigour of qualitative studies, we followed the four criteria proposed by Lincoln and Guba: credibility, transferability, dependability and confirmability. ${ }^{25}$

\section{Procedures}

Data were collected in July 2015. For verifying the accuracy, the data were coded and categorised independently by two authors, and the emerged themes were compared. ${ }^{23}$ To provide transferability, participants varied in gender, age, educational level, occupation, duration of diabetes and internet use. The data recorded from each face-to-face interview were quickly transcribed and analysed immediately after the interview to obtain information on data saturation, which was achieved with nine patients, and dependability. This study was limited to two research questions; hence, the analysis quickly yielded data that did not add new information to the number of categories found, which suggested data saturation. The transcribed raw data of all interviews were read through several times to produce an overall general impression and field notes were made during interviews.

The questions were based on the Persian Diabetes Self-Management Education (PDSME) programme ${ }^{26}$ and educational needs of T2D for self-care ${ }^{27}$ and pilot tested with one patient. Through the interviews, the researcher explored which structure, components and features of internet-enabled instructional design should be suitable for patients with DM, besides the obstacles and benefits of using new technology.

An inductive thematic analysis ${ }^{28}$ was used to identify themes describing the educational needs of patients with DM and design features of internet-enabled education. In the first step, all interviews were read and reread as whole entities to better understand the data. Ideas or patterns of interest were marked in the text. The second step involved identifying and coding meaningful groups of text. Thereafter, the different codes were collated into categories and subsequently into potential themes. Finally, the themes were reviewed in relation to the codes, and the specifics of each theme and the overall narrative were refined. During the analysis, the entire data set, the coded meaningful groups of text and the ongoing analysis of the data were iteratively performed. The entire analysis process and consensus were conducted by two of the authors supported by the whole research team through discussions to confirm the consistency of the findings.

\section{Ethical considerations}

All participants received oral and written information about the study and read and signed a consent form. Participants were assured confidentiality of the gathered data as well as anonymity. They were also informed of their right to withdraw from the study at any time.

\section{RESULTS}

The nine participants included five men and four women with a mean age of 43.3 years, and the average number of years since being diagnosed was 7.8 years. The participants had been using the internet on average for 7.6 years.

\section{Data analysis}

We derived 207 codes from the interviews; these resulted in 38 subcategories, 10 categories and 4 themes. Categories and themes are shown in table 1.

\section{(1) Seeking knowledge about diabetes}

Seeking knowledge about diabetes was the theme to which patients contributed the most. This theme indicated the types of learning requirements, technology-allowed interaction and the acquisition of knowledge about general and specific diabetes issues that the patients regarded as important to include in internet-enabled patient education. Participants indicated that gaining awareness about diabetes and how to better control blood sugar are important matters to them.

Table 1 Categories and themes derived from the interview data

\begin{tabular}{ll}
\hline Categories & Themes \\
\hline Requirements for learning & (1) Seeking knowledge about diabetes \\
Acquisition of knowledge & \\
Specific knowledge acquisition & \\
Interaction & (2) Teaching and learning \\
Teaching methods & (3) Facilitators \\
Different ways to learn & \\
Facilitators for using internet & (4) Barriers \\
Facilitators for using mobile & \\
Barriers for using internet & Perceived cultural barriers
\end{tabular}


To know more is better, especially about food intake. I think that if I know more it is much better for me, and the most important thing to know is about eating behaviour. Patient 9, male, 23 years

A number of participants asked to have access to and find information about diabetes by using new technologies, such as smart phones, in order to receive updated information.

I feel that the information should always be available. For example, via mobile phone or SMS is a good way. Patient 8 , male, 44 years

Other participants insisted on getting more information, for example, about diet, stress and drug complications in their daily life. Diet was a major concern for many patients, and they did not know how they could control their regimen. Stress was also a main issue, and they wanted to know how they could control or decrease stress.

I'd like to know which things can lower the blood sugar and what kind of food I can eat. Patient 2, female, 44 years

I would like to know more about stress, and I think I have a lot of stress because of my disease. Why do I have this stress? I fear eating food, and why do I have this fear? Patient 7 , female, 52 years

I would like to know what the side effects are from medications that we take and what their long-term side effects are on the body. Patient 7 , female, 52 years

Participants preferred to having access to consultation through technology and getting help from others, especially physicians. Most of them did not find a Persian website about diabetes, and they noted how helpful it would be if one existed.

My doctor told me something useful about nutrition that had a good influence on me. He told me that I should eat less and try to eat snacks and fruit. Patient 5, male, 40 years

If a Persian website were available, I would definitely use it, but I do not know whether one is available. Patient 9, male, 23 years

\section{(2) Teaching and learning}

The teaching and learning theme included using different teaching methods and different ways to learn about diabetes that should be included in online patient education. The participants showed interest in getting educational material to read and learn about diabetes, both in formal educational classes and also through a 'question and answer' feature with a direct communication with doctors and peers.

I think the internet is a good option if we can have a specific website that could allow us to ask questions about our problem and also communicate with physicians and other patients who have the same problem. Patient 8 , male, 44 years

The participants suggested that they would like to get information on diabetes by watching videos, reading educational material and receiving text messages. Participants stated that they would like to have pretests and post-tests of the educational material they read to test their understanding. However, they also stated that receiving too much information on diabetes, possibly via text messages, could be irritating.

Best of all is to watch educational movies, questions and answers. Communication between other patients and physician is also good. Patient 1 , male, 56 years

SMS is an effective way, and three times per week is good. Once a week may be forgotten, and every day could be boring. One should consider the psychological aspects of its effect. Patient 6 , male, 58 years

I do not read a lot but I try to watch and listen since I believe I remember better. Patient 3 , female, 26 years

Some of the participants had experience in browsing the internet and could find helpful information, but they did not find information in Persian about diabetes. They also preferred to get updates about the disease.

I like to search the web and see what is good for diabetic patients. So far I have read on the internet, and I know there is good information but it is not in Persian. Patient 2 , female, 44 years

If new information about diabetes comes up, then notification by SMS is a good thing. New information via email is also good, but rarely do people check their email. Patient 3, female, 26 years

SMS should be sent only when there is something new that they [the caregivers] want to tell us. Patient 3, female, 26 years

\section{(3) Facilitators}

Facilitators to patient education were the internet and mobile phones, as expressed by the participants. They also mentioned facilitating characteristics, such as welldesigned, easy to use, well optimised for search, quick startup time, responsiveness and a focused purpose. The participants welcomed an Iranian educational website.

If we could have a site that patients could have access to, like "Iran Diabetes Association" site, it would be very good, but it does not exist. Patient 6 , male, 58 years

It is good to use the mobile phone with interesting content about diabetes that could increase life expectancy. I do not like today's technology because we are missing a lot of things, like human contact and being together, but unfortunately we should accept them 
because they became a part of every day's life. Patient 3 , female, 26 years

It would be nice if we could ask questions to solve our problems because sometimes we simply don't have access to our physician. Patient 3, female, 26 years

I think the internet is good for patients as long as we can have access to a specific [diabetes] website quickly in order to ask questions and add my comments and also to communicate with other patients. Patient 8, male, 44 years

\section{(4) Barriers}

The barriers to internet-enabled patient education, as stated by the participants, included proper lack of internet access, uncertainty of connectivity to the internet and lack of a proper Persian site. The participants also perceived cultural barriers, such as fears about the internet, as well as a lack of time and awareness.

I have not seen any comprehensive website in Persian. I like a reputable site that my doctor confirms; this is very important to me. Patient 3, female, 26 years

I am not sure about the content on the internet because it is different from one site to another. For example, about herbal medicine and eating fruit, I don't know which fruit has sugar and I don't know how much watermelon I should eat.' Patient 8 , male, 44 years

I did not search the internet about diabetes because I did not have the time. I have had the internet for 10 years but was diagnosed three years ago with diabetes. Patient 9 , male, 23 years

Participants reported about the variation in information and content that is available on the internet as well as opposing views of doctors.

There is a lot of information about diabetes on websites, but when I look at them they have several perspectives in eating habits, yet the physician's treatment is the same. Patient 8 , male, 44 years

Some participants reported of having access to the internet and mentioned that using the internet and computers are not popular yet with the Iranian population.

All [Iranian] people do not have access to the internet, and some of them do not like having a computer or laptop. Patient 1 , male, 56 years

Internet access is not widely available and [on-line] accessibility to a diabetes physician is hard. Patient 8, male, 44 years

There were some cultural barriers that participants mentioned, such as lack of awareness and lack of time to think about diabetes, as well as physicians not taking their time to talk to patients and educate them.
I am afraid to eat food that I do not know if it is ok for me to eat. I am afraid of insulin. I do not know where this fear comes from; perhaps it is because one of my relatives died of diabetes at a young age, and he was using insulin. Patient 7, female, 52 years

I do not know what my blood glucose should be. I asked one time and they told me 94 to 110 is good, and I do not know if I have diabetes type one or two. I need information. Patient 8 , male, 44 years

Doctors do not have time to give information that patients need. In Iran, physicians look like businessmen, and unfortunately they do not have time for patients. Patient 8 , male, 44 years

\section{DISCUSSION}

In this study, we explored the educational needs and design features of personalised web-based and mobile education with a sample of Iranian patients with DM. Through interviews, four themes emerged from the data. The first theme was seeking knowledge about diabetes, and it included specific knowledge acquisition, patient's interactions and learning requirements. The second theme was teaching and learning and it included using different teaching methods and different ways to learn about the disease. The third theme was facilitators, and it included features of internet and use of mobile phone to learn about the disease. The last theme was barriers, and it included the lack of proper internet access and a local language website about diabetes. Between barriers there were also perceived cultural barriers, such as patient's uncertainty about the internet, lack of time and awareness.

Patients' educational and technological needs are flexible and specific at the same time. As another study has shown, ${ }^{29}$ the participants prefer educational material that is comprehensive and well organised and want access to what they want to know and when they need to know it; this is thought to be helpful for confident decision-making. Supplemental information should be produced with cultural sensitivity and an appropriate literacy level.

The results of our study highlight the importance of patients receiving standardised general diabetes education with a specific focus on dietary interventions and the importance of physical activity, which has direct relevance to clinical outcomes. ${ }^{6}$

Although not prevalent as communication tools for patients in Iran, internet and mobile phones can act as facilitators, as expressed by the participants; especially when they have certain features, such as being welldesigned, easy to use, well optimised for searches, a quick startup time, responsive and with a focused purpose. The results of this study partially confirm a review study ${ }^{30}$ that showed data export and communication are some of the most common features of mobile phone diabetes apps. However, the same study noted 
that personalised education is an under-represented feature. The participants particularly welcomed a local language educational website.

There are a large number of diabetes apps that have been developed and available, but many of them are not suited to one's needs and a number of those lack proper usability and needs orientation. ${ }^{18}$ In this study, the researcher tried to identify the educational and technical needs of the target group in order to prepare internet-enabled services that can help improve the patients' self-care management of the disease.

The results confirmed that there is a mix of barriers to patient education, including what can be interpreted as an inadequate health system with challenging communication interfaces that make it difficult, for patients with DM, to use internet-enabled technology. In addition, low health literacy or inability to identify the need and health information resources results in difficulty to apply disease-related knowledge in daily life. This is an important barrier that has been previously addressed; as researchers have expressed, there is no doubt that development of educational programmes is the least expensive and the most practical way to meet consumers of health. ${ }^{31}$ Some studies have shown other barriers to using digital tools for diabetes management, including cost, insufficient scientific evidence, not useful in certain populations, data protection and data security. ${ }^{19}$ Despite many barriers, the digital industry is growing rapidly, and it is estimated that in the future all electronic health record systems will be integrated using a common platform. ${ }^{19}$

Research in England shows that only $6.0 \%$ of all people with T2D have been offered a diabetes selfmanagement educational programme that could help them take suitable action against the chronic disease. ${ }^{15}$ In Iran, based on the PDSME, ${ }^{26}$ researchers will offer and use this educational programme by making use of new technology for improving the daily life of people with diabetes.

Our study demonstrates that despite the significant amount of work needed in Iran to understand patients' needs and to design technology to best serve these needs, new technology can serve as a catalyst for change. Research continues to provide evidence that an online diabetes self-management programme is acceptable for patients with $\mathrm{DM}^{32}$ and that mobile phones can help improve self-management behaviour. ${ }^{33}$

The results of this research have identified patienttailored information, interactivity, content credibility, clear presentation of content, use of multimedia and language contextualisation as essential design features of technology directed at patient education of chronic disease management in an Iranian population. Our findings confirm those of earlier research. ${ }^{34}$

\section{Limitations}

A limitation of this study is that the sample was selected from one diabetes clinic in a large city, and therefore the findings may not be transferable to a broader population with diabetes. However, our results are useful for middle-income and middle-eastern countries, which have a similar culture, similar problems for patients with $\mathrm{DM}$ and similar problems with technology penetration.

\section{CONCLUSION}

The patients' needs and technology design features resulting from this research are essential for designing functional mock-ups of a personalised diabetes education that the researchers will use in the next phase of the study. Through design-based research, the researchers will design internet-enabled educational material for self-care management of patients with DM in Iran. Our objective is to reach patients with DM with empirically designed internet-enabled learning services that are clinically relevant and that can help improve the patients' self-care management of the disease.

\section{Author affiliations}

${ }^{1}$ Department of Learning, Informatics, Management and Ethics, Karolinska Institutet, Stockholm, Sweden

${ }^{2}$ Education Development Center, Mashhad University of Medical Sciences, Mashhad, Iran

${ }^{3}$ Evidence-Based Caring Research Center, Mashhad University of Medical Sciences, Mashhad, Iran

${ }^{4}$ Department of Medical-Surgical Nursing, School of Nursing and Midwifery, Mashhad University of Medical Sciences, Mashhad, Iran

${ }^{5}$ Department of Medical Education, School of Medicine, Mashhad University of Medical Sciences, Mashhad, Iran

${ }^{6}$ Lee Kong Chian School of Medicine, Nanyang Technological University, Singapore, Singapore

${ }^{7}$ Department of Clinical Science and Education, Södersjukhuset, Karolinska Institutet, Stockholm, Sweden

Twitter Follow Nabil Zary at @nzary

Acknowledgements The authors wish to thank the Vice Chancellor of Mashhad University of Medical Sciences who supported this study. They thank all participants and staff at the Mashhad Diabetes Clinic for their cooperation in conducting this research.

Contributors JJ conceived and designed the study, obtained funding, conducted interviews and analysed the data, drafted the manuscript and prepared the tables. HKM conducted data analysis and edited the manuscript. NZ assisted in the design of the study and edited the manuscript. IM assisted in the design of the study, drafted and edited the manuscript. All authors were involved in the writing of the manuscript.

Funding This research is funded by the Vice Chancellor of Research at Mashhad University of Medical Sciences (grant number 940757).

Competing interests None declared.

Patient consent Obtained.

Ethics approval The study was approved by the Ethical Committee of Mashhad University of Medical Sciences (number IR.MUMS.REC.1395.108).

Provenance and peer review Not commissioned; externally peer reviewed.

Data sharing statement No additional data are available.

Open Access This is an Open Access article distributed in accordance with the Creative Commons Attribution Non Commercial (CC BY-NC 4.0) license, which permits others to distribute, remix, adapt, build upon this work noncommercially, and license their derivative works on different terms, provided the original work is properly cited and the use is non-commercial. See: http:// creativecommons.org/licenses/by-nc/4.0/ 


\section{REFERENCES}

1. International Diabetes Federation. IDF Diabetes Atlas 7th Edition Brussels, Belgium. idf.org. 2015:1-4. http://www.diabetesatlas.org (accessed 25 Jun 2016).

2. Tobias M. Global control of diabetes: information for action. Lancet 2011;378:3-4.

3. Whiting DR, Guariguata L, Weil C, et al. IDF Diabetes Atlas: globa estimates of the prevalence of diabetes for 2011 and 2030. Diabetes Res Clin Pract 2011;94:311-21.

4. Schulze MB, Hu FB. Primary prevention of diabetes: what can be done and how much can be prevented? Annu Rev Public Heal 2005;26:445-67.

5. Garber AJ, Abrahamson MJ, Barzilay Jl, et al. American Association of Clinical Endocrinologists' Comprehensive Diabetes Management Algorithm 2013 Consensus Statement. Endocr Pract 2013;19:1-48.

6. Inzucchi SE, Bergenstal RM, Buse JB, et al. Management of hyperglycaemia in type 2 diabetes, 2015: a patient-centred approach. Update to a Position Statement of the American Diabetes Association and the European Association for the Study of Diabetes. Diabetologia 2015;58:429-42.

7. Agrimon $\mathrm{OH}$, Street J, Info A. Developing comprehensive diabetes education materials for structured patient education programs in primary care setting. Int J Public Heal Sci 2016;5:16-26.

8. Wong $\mathrm{C}$, Harrison $\mathrm{C}$, Britt $\mathrm{H}$, et al. Patient use of the internet for health information. Aust Fam Physician 2014;43:875-7.

9. Hanley J, Fairbrother P, McCloughan L, et al. Qualitative study of telemonitoring of blood glucose and blood pressure in type 2 diabetes. BMJ Open 2015;5:e008896.

10. Farmer A, Wade A, Goyder E, et al. Impact of self monitoring of blood glucose in the management of patients with non-insulin treated diabetes: open parallel group randomised trial. BMJ 2007;335:132.

11. Chen CM, Chang Yeh M. The experiences of diabetics on self-monitoring of blood glucose: a qualitative metasynthesis. J Clin Nurs 2015;24:614-26.

12. Polisena J, Tran K, Cimon K, et al. Home telehealth for diabetes management: a systematic review and meta-analysis. Diabetes Obes Metab 2009;11:913-30.

13. Jones H, Edwards L, Vallis TM, et al. Changes in diabetes self-care behaviors make a difference in glycemic control: the diabetes stages of change (DiSC) study. Diabetes Care 2003;26:732-7.

14. Steventon A, Bardsley M, Doll H, et al. Effect of telehealth on glycaemic control: analysis of patients with type 2 diabetes in the Whole Systems Demonstrator cluster randomised trial. BMC Heal Serv Res 2014:14:334.

15. Huxley C, Sturt J, Dale J, et al. Is it possible to predict improved diabetes outcomes following diabetes self-management education: a mixed-methods longitudinal design. BMJ Open 2015;5:e008781.

16. Shetty AS, Chamukuttan S, Nanditha A, et al. Reinforcement of adherence to prescription recommendations in Asian Indian diabetes patients using short message service (SMS) - a pilot study. J Assoc Physicians India 2011;59:711-14.

17. Quinn CC, Clough SS, Minor JM, et al. WellDoc (TM) mobile diabetes management randomized controlled trial: change in clinical and behavioral outcomes and patient and physician satisfaction. Diabetes Technol Ther 2008;10:160-8.

18. Arnhold M, Quade M, Kirch W. Mobile applications for diabetes: a systematic review and expert-based usability evaluation considering the special requirements of diabetes patients age 50 years or older. J Med Internet Res 2014;16:e104.

19. Shah VN, Garg SK. Managing diabetes in the digital age. Clin Diabetes Endocrinol 2015;1:16.

20. Miniwatts Marketing Group. Internet Users in 2015 in the Middle East and the World. Internet World Stats. 2015:3-6. http://www. internetworldstats.com/stats5.htm\#me (accessed 23 Jun 2016).

21. Jafari J, karimi Moonaghi $\mathrm{H}$, Ahmady S, et al. Readiness of diabetics to use the internet and mobile services: the case of a large city in a middle-income country. J Community Med Health Educ 2015;5.

22. Dolmans DHJM, Tigelaar D. Building bridges between theory and practice in medical education using a design-based research approach: AMEE Guide No. 60. Med Teach 2012;34:1-10.

23. Elo $\mathrm{S}$, Kyngäs $\mathrm{H}$. The qualitative content analysis process. J AdV Nurs 2008;62:107-15.

24. Tong A, Sainsbury $P$, Craig J. Consolidated criteria for reporting qualitative research (COREQ): a 32- item checklist for interviews and focus group. Int J Qual Heal Care 2007;19:349-57.

25. Denzin NK, Lincoln YS. The SAGE handbook of qualitative research. The SAGE Handbook, 2011:563-681.

26. Shakibazadeh E, Bartholomew LK, Rashidian A, et al. Persian Diabetes Self-Management Education (PDSME) program: evaluation of effectiveness in Iran. Health Promot Int 2016;31:623-34.

27. Haas L, Maryniuk M, Beck J, et al. National standards for diabetes self-management education and support. Diabetes Care 2014;37 (Suppl 1):S144-53.

28. Becker SI. Guidance of attention by feature relationships: the end of the road for feature map theories? Curr Trends Eye Track Res 2014:3:37-49.

29. Garshasbi S, Khazaeipour Z, Fakhraei N, et al. Evaluating knowledge, attitude and practice of health-care workers regarding patient education in Iran. Acta Med Iran 2016;54:58-66.

30. Chomutare T, Fernandez-Luque L, Arsand E, et al. Features of mobile diabetes applications: review of the literature and analysis of current applications compared against evidence-based guidelines. J Med Internet Res 2011;13.

31. Nikbakht Nasrabadi A, Sabzevari S, Negahban Bonabi T. Iranian women's experiences of health information seeking barriers: a qualitative study in Kerman. Iran Red Crescent Med J 2015;17: e25156.

32. Lorig K, Ritter PL, Laurent DD, et al. Online diabetes self-management program: a randomized study. Diabetes Care 2010;33:1275-81.

33. Krishna S, Boren SA. Diabetes self-management care via cell phone: a systematic review. J Diabetes Sci Technol 2008;2:509-17.

34. Win KT, Hassan NM, Oinas-Kukkonen $\mathrm{H}$, et al. Online patient education for chronic disease management: consumer perspectives. J Med Syst 2016;40:1-13. 